\title{
The effect of saliva stimulation on the secretion of cortisol during stress and physiological conditions
}

\author{
Jansakova K, Kyselicova K, Celusakova H, Repiska G, Ostatnikova D \\ Institute of Physiology, Faculty of Medicine, Comenius University, Bratislava, Slovakia. \\ jansakova.katarina@gmail.com
}

\begin{abstract}
OBJECTIVES: Many studies use stimulated saliva for the assessment of cortisol. However, it is not yet clear how stimulation affects the flow of specific markers. The aim was to assess whether stimulation of salivation affects the physiological flow of cortisol during a stressing day as compared to an ordinary day. The second aim was to show how the normalising factor affects the outcome of the study.

METHODS: Stimulated saliva was taken from 42 children at 8:00 a.m. and 12:00 a.m. on two separate days one month apart. During the first day, the children were exposed to stress situation, while the second day was considered a control day. The concentration of cortisol was analysed using ELISA.

RESULTS: The highest level of cortisol was observed in the morning of the stress day $(p<0.001)$. The correction of the result for the salivary protein concentration showed no significant differences between morning and afternoon cortisol levels on both the testing $(p=0.87)$ and control days $(p>0.99)$.

CONCLUSION: Based on our results, the examination of the cortisol diurnal rhythm is not reliable in stimulated saliva. Moreover, the effect of saliva stimulation has to be taken into account for every marker individually (Fig. 2, Ref. 22). Text in PDF www.elis.sk.

KEY WORDS: cortisol, saliva, stimulation.
\end{abstract}

\section{Introduction}

Cortisol is liable to diurnal rhythm. Its physiological concentration may vary depending on the exposure to stress stimuli (1), physical activity (2) or presence of a disease. Cortisol is quantifiable in various biological samples including saliva. (3). Saliva can be obtained easily, non-invasively and repeatedly. However, the collection of saliva might be difficult in some categories of studied subjects such as young children, older people, or individuals with salivary gland diseases (4-6). Therefore, the stimulation of salivation may be a good way for getting a higher volume of saliva (7). On the other hand, the composition of stimulated saliva might be changed in comparison to unstimulated saliva (8). It was found that the concentration of salivary cortisol is influenced by the salivary stimulant (6). Kidd et al (9) reported no difference in cortisol concentration in unstimulated vs citric acid-stimulated saliva but there was a significant difference in unstimulated saliva vs saliva collected by swabs.

The main problem is that the majority of studies use stimulated saliva $(10,11)$, thus, different studies can get different data

Institute of Physiology, Faculty of Medicine, Comenius University, Bratislava, Slovakia

Address for correspondence: $\mathrm{K}$. Jansakova, MSc, PhD, Institute of Physiology, Faculty of Medicine Comenius University, Sasinkova 2, SK-813 72 Bratislava, Slovakia. Phone: +421.2 .90119516$

Acknowledgement: This study was supported by the Slovak Research and Development Agency grant number APVV 15-0045 and APVV 15-0085. leading to their different interpretations (6). However, all these complications are not usually considered and studies pointing to the complications regarding the collection of saliva are missing.

The aim of this study was to analyse if the stimulation of salivation affects the diurnal cortisol flow during exposure to psychological stress on the day of testing compared to the control day without stress strain.

\section{Material and methods}

The study was approved by the Ethics Committee of the Comenius University Faculty of Medicine and the University Hospital in Bratislava, Slovakia and complies with the 1964 Helsinki declaration and its later amendments.

\section{Design of the study}

A total number of 42 healthy children $(10.82 \pm 0.08,11$ girls, 31 boys) were recruited. Stimulated saliva was collected into a sterile tube using visual stimuli - a lemon. The collection of saliva was performed at 8:00 a.m. and 11:30 a.m. during two separate days one month apart. On the first day, the children were exposed to stress situation represented by test questions evaluating their general competences in mathematics, natural sciences, mother tongue and foreign language. The second day represented a control day to the testing day with no stress activity and mental strain. The design of the experiment is described in Figure 1.

Saliva samples were kept on ice and, after their delivery to the laboratory, were centrifuged at $1,000 \mathrm{~g}$ for 10 minutes. The par- 


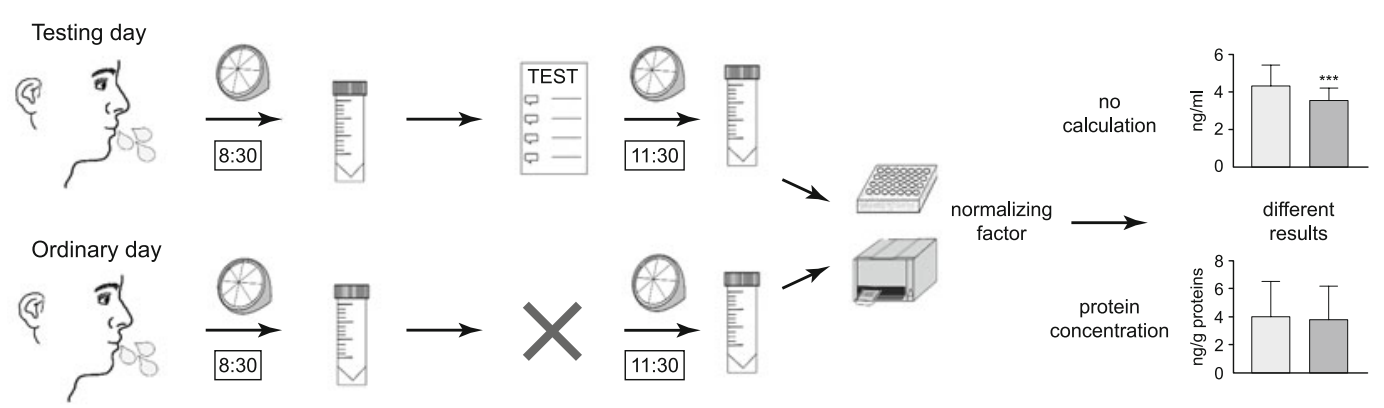

Fig. 1. Design of the study.

ticipants were asked not to eat or drink flavoured beverages one hour before saliva collection.

\section{Salivary cortisol analysis}

Salivary cortisol level was measured using the salivary cortisol ELISA kit (DRG instruments GmbH, Marburg, Germany) according to manufacturer's instructions. The concentration of salivary proteins was assessed using BCA kit (Sigma-Aldrich, Munich, Germany).

\section{Statistical analysis}

For statistical analysis, GraphPad Prism 7 software was used (GraphPad Software, Inc., La Jolla, CA, USA). Data were evaluated using repeated measures with two-way ANOVA and Tukey post hoc test. The statistical significance was set to $p=0.05$. The results are presented as mean + standard deviation.

\section{Results}

The visual stimulation affected the cortisol flow on the ordinary day. The highest level of salivary cortisol was observed in the morning of the testing day (Fig. 2A). There was a significant difference between morning and afternoon cortisol levels on the testing day $(\mathrm{p}<0.001)$, and morning $(\mathrm{p}=0.003)$ and afternoon ( $p$ $<0.001)$ cortisol levels on the ordinary day. No significant differ-

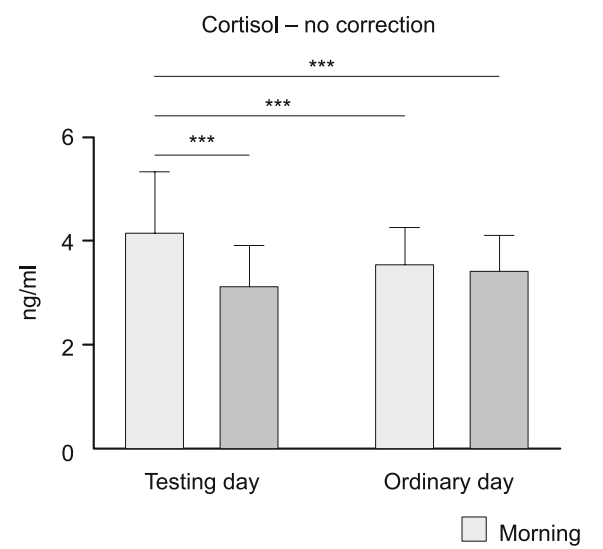

ences were observed between the afternoon cortisol levels on the testing and ordinary days $(\mathrm{p}=0.89)$.

The correction of results for total protein concentration of saliva (Fig. 2B) showed no significant differences between individual days. No significant differences were observed between the morning and afternoon values on both testing $(p=0.87)$ and ordinary days $(\mathrm{p}>0.99)$. There was even no difference between morning cortisol levels on testing and ordinary days $(p=0.95)$.

\section{Discussion}

Many studies point to the usability and applicability of saliva in relation to the detection and potential diagnosis of oral and systemic diseases or various disorders (12-14). All these studies were focused on the reliable use of saliva in clinical practise. Unfortunately, there is still a long way ahead due to several complications considering saliva as diagnostic fluid equal to blood. One of the most important factors influencing its application is the salivary collection process. There are two major ways of how to collect saliva, namely unstimulated and stimulated, while both have their own pros and cons. Several studies showed that the stimulation of salivation affects the composition of saliva and, thus, directly affects the result of individual analyses $(6,15,16)$.

In this study we focused on the effect of visually stimulated saliva on cortisol during the stress day as opposed to resting day with-

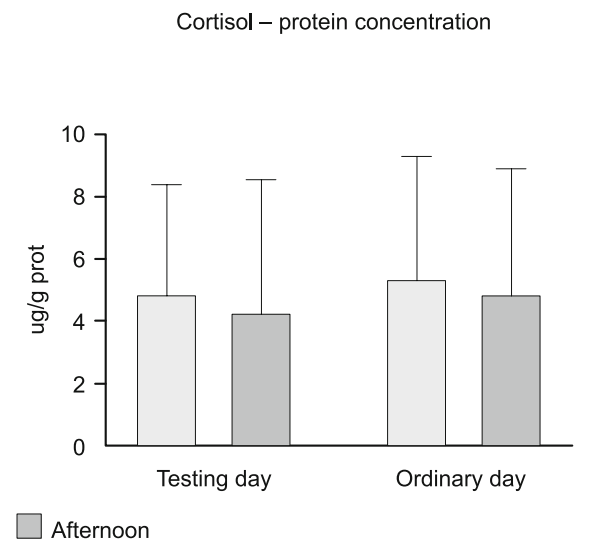

Fig. 2. Salivary cortisol: Cortisol assessment in stimulated saliva collected in the morning and afternoon of the testing and ordinary days A) without further calculations and $\mathrm{B}$ ) correction of the results for protein concentration; $* *$ denotes $\mathbf{p}<0.01$, $* * *$ denotes $\mathbf{p}<0.001$; data are presented as mean + standard deviations. 
out stress strain. Next, we point to the necessity of a normalizing factor and its impact on the results and overall output of the study.

Psychological stress increased salivary cortisol concentrations as expected. The absence of the stress factor during the control day caused clearance of differences between morning and afternoon cortisol level in stimulated saliva. Under physiological conditions, the stimulation of salivation probably leads to a continual secretion of cortisol into saliva regardless of daytime. No difference was observed even between afternoon saliva samples taken on the stress day and morning saliva collected on the ordinary day. Contrary to our findings, there are a lot of published studies showing that the stimulation of salivation had no effect on cortisol diurnal rhythm. Moreover, they observed gradually decreasing cortisol concentrations during the day $(17,18)$. In addition, salivary studies are, in general, accompanied by high variability even in unstimulated saliva (19). This problem may be solved or at least minimalized by using the normalizing factor. However, using the normalizing factor may affect the overall outcome of the study. The cortisol concentration was additionally calculated for total salivary protein concentration. We observed that natural non-corrected data differed from corrected ones. Non-corrected data showed that the highest level of cortisol is in the morning of the stress day. However, the correction of results for protein concentration wipes off this difference between the collected samples.

This fact points to the necessity of optimizing the pre-analytical (collection) and post-analytical phase (normalizing factor) procedure for every marker individually $(6,20)$. Every type of collection and its effect ought to be defined for every salivary marker.

Also, several other facts should be considered when using saliva, e.g., oral health. Increased levels of cortisol were observed in saliva of patients with burning mouth syndrome (21) and children with dental caries (22).

The main limitation of this study is that saliva samples were not collected throughout the whole day until late evening. Even though it is not the purpose of this study, one of the limitations is the missing collection of unstimulated saliva. The comparison of stimulated and unstimulated saliva would show that even visual stimulation changes the composition of saliva. The next limitation is that the salivary flow rate of stimulated saliva was not recorded. Unfortunately, we were not able to adjust our results on flow rate and compare them with those normalized on protein concentration.

To conclude, using stimulated saliva for monitoring of standard physiological concentration of markers liable to diurnal rhythm like cortisol should be considered.

\section{References}

1. Gordis EB, Granger DA, Susman EJ, Trickett PK. Asymmetry between salivary cortisol and $\alpha$-amylase reactivity to stress: Relation to aggressive behavior in adolescents. Psychoneuroendocrinology 2006; 31 (8): 976-987.

2. Tauler P, Martinez S, Moreno C, Martinez P, Aguilo A. Changes in salivary hormones, immunoglobulin $\mathrm{A}$, and $\mathrm{C}$-reactive protein in response to ultra-endurance exercises. Appl Physiol Nutr Metab 2014; 39 (5): 560-565.

3. El-Farhan N, Rees DA, Evans C. Measuring cortisol in serum, urine and saliva - are our assays good enough? Ann Clin Biochem 2017; 54 (3): 308-322.
4. Nagler RM, Hershkovich O. Age-related changes in unstimulated salivary function and composition and its relations to medications and oral sensorial complaints. Aging Clin Exp Res 2005; 17 (5): 358-366.

5. Osailan SM, Pramanik R, Shirlaw P, Proctor GB, Challacombe SJ. Clinical assessment of oral dryness: development of a scoring system related to salivary flow and mucosal wetness. Oral Surg Oral Med Oral Pathol Oral Radiol 2012; 114 (5): 597-603.

6. Granger DA, Kivlighan KT, Fortunato C, Harmon AG, Hibel LC, Schwartz EB et al. Integration of salivary biomarkers into developmental and behaviorally-oriented research: problems and solutions for collecting specimens. Physiol Behav 2007; 92 (4): 583-590.

7. Feltsan T, Stanko P, Mracna J. Sjogren s syndrome in present. Bratisl Lek Listy 2012; 113 (8): 514-516.

8. Gittings S, Turnbull N, Henry B, Roberts CJ, Gershkovich P. Characterisation of human saliva as a platform for oral dissolution medium development. Eur J Pharm Biopharm 2015; 91: 16-24.

9. Kidd S, Midgley P, Lone N, Wallace AM, Nicol M, Smith J et al. A reinvestigation of saliva collection procedures that highlights the risk of potential positive interference in cortisol immunoassay. Steroids 2009; 74 (8): 666-668.

10. Kobayashi H, Miyazaki Y. Distribution characteristics of salivary cortisol measurements in a healthy young male population. J Physiol Anthropol 2015; 34: 30 .

11. Lai JCL, Leung MOY, Lee DYH, Lam YW, Berning K. Loneliness and Diurnal Salivary Cortisol in Emerging Adults. Int J Mol Sci 2018; 19 (7).

12. Banasova L, Kamodyova N, Jansakova K, Tothova L, Stanko P, Turna $\mathbf{J}$ et al. Salivary DNA and markers of oxidative stress in patients with chronic periodontitis. Clin Oral Investig 2015; 19 (2): 201-207.

13. Said HS, Suda W, Nakagome S, Chinen H, Oshima K, Kim S et al. Dysbiosis of salivary microbiota in inflammatory bowel disease and its association with oral immunological biomarkers. DNA Res 2014; 21 (1): 15-25.

14. Farah R, Haraty H, Salame Z, Fares Y, Ojcius DM, Said Sadier N. Salivary biomarkers for the diagnosis and monitoring of neurological diseases. Biomed J 2018; 41 (2): 63-87.

15. Beltzer EK, Fortunato CK, Guaderrama MM, Peckins MK, Garramone BM, Granger DA. Salivary flow and alpha-amylase: collection technique, duration, and oral fluid type. Physiol Behav 2010; 101 (2): 289-296.

16. Kamodyova N, Celec P. Salivary markers of oxidative stress and Salivette collection systems. Clin Chem Lab Med 2011; 49 (11): 1887-1890.

17. Nagamine M, Noguchi H, Takahashi N, Kim Y, Matsuoka Y. Effect of cortisol diurnal rhythm on emotional memory in healthy young adults. Sci Rep 2017; 7 (1): 10158.

18. Kjolhede EA, Gustafsson PE, Gustafsson PA, Nelson N. Overweight and obese children have lower cortisol levels than normal weight children. Acta Paediatr 2014; 103 (3): 295-299.

19. Lettrichova I, Tothova L, Hodosy J, Behuliak M, Celec P. Variability of salivary markers of oxidative stress and antioxidant status in young healthy individuals. Redox Rep 2015.

20. Mohamed R, Campbell JL, Cooper-White J, Dimeski G, Punyadeera C. The impact of saliva collection and processing methods on CRP, IgE, and Myoglobin immunoassays. Clin Transl Med 2012; 1 (1): 19.

21. Kim HI, Kim YY, Chang JY, Ko JY, Kho HS. Salivary cortisol, 17beta-estradiol, progesterone, dehydroepiandrosterone, and alpha-amylase in patients with burning mouth syndrome. Oral Dis 2012; 18 (6): 613-620.

22. Caruso S, Gatto R, Cinque B, Cifone MG, Mattei A. Association between salivary cortisol level and caries in early childhood. (1591-996X (Print)).

Received February 4, 2020. Accepted April 9, 2020. 